Pacific Journal of Mathematic 


\section{HOMOMORPHISMS ON NORMED ALGEBRAS}

\section{BerTram YoOD}

1. Introduction Let $B_{1}$ and $B$ be real normed $Q$-algebras (not necessarily complete) and $T$ be a homomorphism of $B_{1}$ into $B$. Our main object is to show that, for certain algebras $B, T$ will always be either continuous or closed if the range $T\left(B_{1}\right)$ contains "enough" of $B$. If $B$ is the algebra of all bounded linear operators on a Banach space $\mathfrak{X}$ and $T\left(B_{1}\right)$ contains all finite-dimensional operators then $T$ is continuous. If $B$ is primitive with minimal one-sided ideals, $T\left(B_{1}\right)$ is dense in $B$ and intersects at least one minimal ideal of $B$ then $T$ is closed. Other examples are given. In these results we can obtain the conclusion for ring homomorphism as well as algebra homomorphism if we assume that $\rho(T(x)) \leqq \rho(x), x \in B_{1}$, where $\rho(x)$ is the spectral radius of $x$. Note that this is a necessary condition for real-homogeneity. For the application of these results it is desirable to have examples of algebras which are $Q$-algebras in all possible normed algebra norms. Examples are given in $\S 2$. For previous work on the continuity of homomorphisms and the homogeneity of isomorphisms on Banach algebras see [8], [9], [11], [12] and [14].

2. Normed Q-algebras and continuity of homomorphisms. For the algebraic notions used see [6]. Let $B$ be a normed algebra over the real field (completeness is not assumed). As in [8], [11] a complex number $\lambda \neq 0$ is in the spectrum of $x \in B$ if it is in the usual complex algebra spectrum of $(x, 0)$ in the complexification of $B$. If $B$ is already a complex algebra then the spectrum of $x$ in this sense is the smallest set in the complex plane symmetric with respect to the real axis which contains the spectrum of $x$ in the complex algebra sense. Let $\rho(x)$ be the spectral radius of $x, \rho(x)=\sup |\lambda|$ for $\lambda$ in the spectrum of $x$. $B$ is called a $Q$-algebra if the set of quasi-regular elements of $B$ is open. Every regular maximal one-sided or two-sided ideal in a $Q$-algebra is closed. Hence the radical of a $Q$-algebra is closed and so also is any primitive ideal. See $[10 ; 77]$.

2.1. Lemma. For a normed algebra $B$ the following statements are equivalent.

(a) $B$ is a Q-algebra.

(b) $\rho(x)=\lim \left\|x^{n}\right\|^{1 / n}, x \in B$.

(c) $\rho(x) \leqq\|x\|, x \in B$.

Received January 14, 1958. This research was supported in part by the National Science Foundation, research grant NSF-G 2573. 
Suppose (a). Then there exists a number $c>0$ such that $x$ is quasiregular for all $x,\|x\|<c$. Set $k=\left[(1+c)^{1 / 2}-1\right]^{-1}$. Let $x \in B$ and $\lambda=a+b i$ be any complex number $\neq 0$ where $\{\lambda|>k||x|\}$. Then

$$
|\lambda|^{-2}\left\|2 a x-x^{2}\right\| \leqq|\lambda|^{-2}\left(2|\lambda|\|x\|+\|x\|^{2}\right)<2 k^{-1}+k^{-2}<c
$$

This shows that $\rho(x) \leqq k\|x\|$. Thus

$$
\rho(x)=\rho\left(x^{n}\right)^{1 / n} \leqq k^{1 / n}\left\|x^{n}\right\|^{1 / n}
$$

for every positive integer $n$. Letting $n \rightarrow \infty$ we see that $c(x) \leqq \lim$ $\left\|x^{n}\right\|^{1 / n}$. But $\lim \left\|x^{n}\right\|^{1 / n}=\rho\left(x \mid B^{c}\right)$, the spectral radius of $x$ in the completion $B^{c}$ of $B$. Hence $\rho(x) \leqq \rho\left(x \mid B^{c}\right)$. Since $\rho\left(x \mid B^{c}\right) \leqq \rho(x)$, (b) follows. Clearly (b) implies (c). Suppose that (a) is false. Then there exists a sequence $\left\{x_{n}\right\}, x_{n} \rightarrow 0$ where $x_{n}$ is not quasi-regular. Then $\rho\left(x_{n}\right) \geqq 1$ for each $n$ and (c) is false.

Let $\mathfrak{X}$ be a Banach space and let $\mathfrak{F}(\mathfrak{X})$ be the Banach algebra of all bounded linear operators on $\mathfrak{X}$ in the uniform topology. Let $\mathfrak{F}(\mathfrak{X})$ be the ideal of all elements of $\mathfrak{F}(\mathfrak{X})$ with finite dimensional range.

2.2. Lemma. Let $j$ be an idempotent in a normed algebra $B$. Then the non-zero spectrum of an element in $j B j$ is the same whether computed in $j B j$ or $B$.

This is given in $[9 ; 375]$ in the complex case. The real case offers no new difficulty.

2.3. THEOREM. Let $U$ be a ring homomorphism or anti-homomorphism of a normed Q-algebra $B_{1}$ into $\mathfrak{\xi}(\mathfrak{X})$ where $U\left(B_{1}\right) \supset \mathfrak{F}(\mathfrak{X})$ and $\rho[U(V)] \leqq \rho(V), V \in B_{1}$. Then $U$ is continuous.

Suppose that $U$ is not continuous. By the additivity of $U$ (see [2; 54]) there exists a sequence $\left\{T_{n}\right\}$ in $B_{1}$ such that $\left\|T_{n}\right\|_{1} \rightarrow 0$ and $\left\|U\left(T_{n}\right)\right\|$ $\rightarrow \infty$ where $\|T\|_{1}$ is the norm in $B_{1}$ and $\|T\|$ is the usual norm in $\mathfrak{F}(\mathfrak{X})$. Consider any idempotent $J$ of $\mathfrak{F}(\mathfrak{X})$ such that $J \mathfrak{F}(\mathfrak{X})$ is a minimal right ideal of $\mathfrak{S}(\mathfrak{X})$. By the work of Arnold [1] these elements $J$ are the linear operators on $\mathfrak{X}$ of the form $J(x)=x^{*}(x) y$ where $x^{*} \in \mathfrak{X}^{*}, y \in \mathfrak{X}$ and $x^{*}(y)=1$. Let $U(W)=J$ and $U\left(T_{n}\right)=V_{n}$. Since $\left\|W T_{n} W\right\|_{1} \rightarrow 0$ we have, by Lemma 2.1, $\rho\left(W T_{n} W\right) \rightarrow 0$ and therefore $\rho\left(J V_{n} J\right) \rightarrow 0$. By Lemma 2.2 and the Gelfand-Mazur theorem, $\left\|J V_{n} J\right\| \rightarrow 0$. Note that $J V_{n} J(x)=x^{*}(x)$ $x^{*}\left[V_{n}(y)\right] y$. Hence $x^{*}\left[V_{n}(y)\right] \rightarrow 0$. Fix $y \neq 0$ in $\mathfrak{X}$. Then $x^{*}\left[V_{n}(y)\right] \rightarrow 0$ for all $x^{*} \in K=\left\{x^{*} \in \mathfrak{X}^{*} \mid x^{*}(y) \neq 0\right\}$. Let $z^{*} \in \mathfrak{X}^{*}, z^{*}(y)=0$. Since $z^{*}$ can be written as the sum of two elements of $K, x^{*}\left[V_{n}(y)\right] \rightarrow 0$ for all $x^{*} \in \mathfrak{X}^{*}$. Hence $\sup \left\|V_{n}(y)\right\|<\infty$ for each $y \in \mathfrak{X}$. By the uniform boundedness theorem, sup $\left\|V_{n}\right\|<\infty$. This is a contradiction.

2.4. THEOREM. Let $T$ be a ring homomorphism or anti-homomorphism of a normed Q-algebra onto a dense subring of a semi-simple 
finitedimensional normed algebra $B$ where $\rho[T(x)] \leqq \rho(x), x \in B_{1}$. Then $T$ is continuous.

By $[7 ; 698] B$ is strongly semi-simple and so, by Theorem proved below, $T$ is real-homogenous and closed. Let $\|x\|_{1}(\|x\|)$ denote the norm in $B_{1}(B)$. Suppose that $T$ is not continuous. Then there ex ists a sequence $\left\{x_{n}\right\}$ in $B_{1}$ such that $\left\|x_{n}\right\|_{1} \rightarrow 0$ and $\left\|T\left(x_{n}\right)\right\|=1, n=1,2, \cdots$. There exists a subsequence $\left\{y_{n}\right\}$ of $\left\{x_{n}\right\}$ such that $\left\|T\left(y_{n}\right)-w\right\| \rightarrow 0$ for some $w \in B$. Since $\|w\|=1$ we contradict the fact that $T$ is a closed mapping.

A normed algebra $B$ is called a permanent $Q$-algebra if it is a $Q$ algebra in all normed algebra norms. We say that the normed algebra $B$ has the spectral extension property if the spectral radius of $x \in B$ is the same as the spectral radius of $x$ considered as an element of any Banach algebra $B_{1}$ in which $B$ may be algebraically imbedded. Examples of algebras with this property are $B^{*}$-algebras [13] and annihilator Banach algebras [3]. To test if a normed algebra $B$ has this property it is sufficient to consider the completions of $B$ in all possible normed algebra norms.

2.5. LemMA. A normed algebra $B$ is a permanent Q-algebra if and only if $B$ has the spectral extension property.

Let $B$ be a permanent $Q$-algebra, $x \in B$. Then $\lim \left\|x^{n}\right\|^{1 / n}$ has the same value $\rho(x)$, by Lemma 2.1 , for any normed algebra norm for $B$. Thus $B$ has the spectral extension property. If $B$ has the latter property then for any norm $\|x\|, \rho(x)=\lim \left\|x^{n}\right\|^{1 / n}$ and $B$ is a permanent $Q$-algebra by Lemma 2.1 .

2.6. THEOREM. Any two sided ideal $I$ of $\mathfrak{F}(\mathfrak{X})$ where $I \supset \mathfrak{F}(\mathfrak{X})$ and any closed subalgebra $B$ of $(\mathfrak{F}(\mathfrak{X}), B \supset \mathfrak{F}(\mathfrak{X})$ have the spectral extension property.

Let $R$ be any such ideal $I$ or closed subalgebra $B$. Let $\|T\|_{1}$ be a normed algebra norm for $R$ and $\|T\|$ the usual norm. For $T \in R$ let $\rho(T)$ be its spectral radius as an element of $R, \rho_{1}(T)$ as an element of the completion of $R$ in the norm $\|T\|_{1}$ and $\rho_{2}(T)$ as an element $\mathfrak{F}(\mathfrak{X})$. In the ideal case if $U \in R$ has a quasi-inverse $V$ in $\mathfrak{F}(\mathfrak{X})$ then $V \in R$. In every case $\rho(T)=\rho_{z}(T)$.

It is enough to show the identity imbedding of $R$ (with norm $\|T\|_{1}$ ) into $\mathfrak{S}(\mathfrak{X})$ (with norm $\|T\|$ ) is continuous. For then there exists $c>0$, $\|T\| \leqq c\|T\|_{1}, T \in R$, whence

$$
\left\|T^{n}\right\|^{1 / n} \leqq c^{1 / n}\left\|T^{n}\right\|_{1}^{3 / n}
$$

for all positive integers $n$. Consequently $\rho(T) \leqq \rho_{1}(T)$. Since $\rho_{1}(T) \leqq \rho(T)$ we would have $\rho(T)=\rho_{1}(T)$. 
Theorem 2.3 cannot be applied since it is not known a priori that $R$ is a $Q$-algebra in the norm $\|T\|_{1}$. If, however, the imbedding is discontinuous there exists a sequence $\left\{T_{n}\right\}$ in $R$ such that $\left\|T_{n}\right\|_{1} \rightarrow 0$ and $\left\|T_{n}\right\| \rightarrow \infty$. By the arguments of [1], the minimal ideals of $R$ are the same as the minimal ideals of $(\mathfrak{F}(\mathfrak{X})$. For each idempotent generator $J$ of a minimal right ideal of $R, J R J$ is a normed division algebra and hence has a unique norm topology by the Gelfand-Mazur theorem. Since $\left\|J T_{n} J\right\|_{1} \rightarrow 0$ we have $\left\|J T_{n} J\right\| \rightarrow 0$. The remainder of the proof may be handled as in Theorem 2.3.

For a ring $B$ and a subset $A \subset B$ we denote the left (right) annihilator of $A$ by $L(A)(R(A))$. Bonsall and Goldie [4] have considered topological rings called annihilator rings in which for each proper right (left) closed ideal $I, L(I) \neq(0)(R(I) \neq(0))$. We consider the related purely algebraic concept of a modular annihilator ring which is defined to be a ring in which $L(M) \neq(0)(R(M) \neq(0))$ for every regular maximal right (left) ideal. From the standpoint of algebra these rings appear to be a natural class containing $H^{*}$-algebras, etc. In view of what follows it is natural to ask if the two concepts agree for semi-simple normed $Q$-algebras or semi-simple Banach algebras. A affirmative answer would settle an unsolved problem in the theory of annihilator algebras.

2.7. LEMMA. Let $B$ be a semi-simple normed annihilator Q-algebra and $I$ be a closed two-sided ideal in $B$. Then $I$ is a modular annihilator Q-algebra.

Thus if we had affirmative answer to the above question, any closed two-sided ideal of a semi-simple annihilator Banach algebra would also be one. The analogous result is known for dual algebras [7; 690].

Let $M$ be a regular maximal right ideal of $I$. Since $I$ is a $Q$-algebra (as an ideal in $B$ ), $M$ is closed in $B$. Since $L(I)=R(I),([4 ; 159]$ ), $L(I+R(I))=(0)$ so that $I+R(I)$ is dense. The arguments of $[7$; Theorem 2] show that $M$ is a right ideal in $B$. We must show $L(M) \cap I \neq(0)$. Suppose the contrary. Then $I L(M)=(0)$ and $L(M) \subset R(I)=L(I)$. As $M \subset I$, $L(M) \supset L(I)$. Therefore $L(M)=L(I) . \quad R(M) M=(0)$ since it is a nilpotent ideal in $B$. Thus $R(M) \subset L(M)=R(I)$. Then since $R(M) \supset R(I)$ we see that $R(M)=L(M)$. If $x \in L(M+R(M))$ then $x \leqq L(M)=R(M)$ and $x \in L R(M)$. Thus $x^{2}=0$ and, by semi-simplicity and the annihilator property, $M+$ $R(M)$ is dense in $B$. Then $(M+R(M)) I=(M+L(I)) I \subset M$ and $B I \subset M$. Let $j$ be a left identity for $I$ modulo $M$. Then $j x-x \in M, x \in I$ and $j x \in M, x \in I$. Hence $I \subset M$ which is a contradiction.

2.8. Lemma. In a semi-simple modular annihilator ring, every proper right (left) ideal contains a minimal right (left) ideal. A normed 
modular annihilator algebra $B$ has the spectral extension property.

Since the first statement is shown by stripping the arguments of Bonsall and Goldie [4] of all topological connotations, a sketch of the argument is sufficient. As in [4, Lemma 2], if $j$ is not right (left) quasi-regular there exists $x \neq 0$ in $B$ where $x j=x(j x=x)$. The arguments of [4, Theorem 1] show that if $M$ is a regular maximal right (left) ideal of $B$ then $L(M)(R(M))$ is a minimal left (right) ideal generated by an idempotent. Also the left (right) annihilator of a minimal right (left) ideal is a regular maximal left (right) ideal. Consider the socle $K$ of $B$. By the reasoning of [4, Theorem 4], $L(K)=R(K)=(0)$. Let $I$ be a proper right ideal of $B$. If $I$ contained no minimal right ideals of $B$ then, as in the proof of [4, Lemma 4], $I \subset L(K)$, which is impossible.

Let $x \in B$ and let $B^{\prime}$ be the completion of $B$ in the normed algebra norm $\|x\|_{1}$. Consider $\lambda=a+b i \neq 0$ in $s p(x \mid B)$. Then $u=|\lambda|^{-2}\left(2 a x-x^{2}\right)$ has no quasi-inverse in $B$. As in [3; p 159] there exists $y \neq 0$ such that $u y=y$ and $u$ has no quasi-inverse in $B^{\prime}$. Then $\rho\left(x \mid B^{\prime}\right)=\rho(x \mid B)$.

3. Closure of homomorphisms and anti-homomorphisms. Throughout this section the following notation is assumed. Let $B_{1}(B)$ be a real normed algebra with norm $\|x\|_{1}(\|x\|) . \quad T$ is a ring homomorphism or anti-homomorphism of $B_{1}$ onto a dense subset of $B . \quad T$ is called closed if $\left\|x_{n}-x\right\|_{1} \rightarrow 0,\left\|T\left(x_{n}\right)-y\right\| \rightarrow 0$ imply that $y \in T\left(B_{1}\right)$ and $y=T(x)$. By the separating set $S$ of $T$ we mean the set of all $y \in B$ such that there exists a sequence $\left\{x_{n}\right\}$ in $B_{1}$ where $\left\|x_{n}\right\|_{1} \rightarrow 0$ and $\left\|y-T\left(x_{n}\right)\right\| \rightarrow 0$. We assume that $\rho[T(x)] \leqq \rho(x), x \in B_{1}$. Note that this condition is automatic if $T$ is real-linear.

The next lemma is an adaptation of results of Rickart [11].

3.1. LEMMA. $T$ is closed and real-homogeneous if and only if $S=(0)$. $S$ is a closed two-sided ideal in $B$ and $T^{-1}(S)$ a closed two-sided ideal in $B_{1}$. If $B_{1}$ is a normed Q-algebra then every element of $S$ is a topological divisor of zero in $B$.

Clearly $T$ is rational-homogeneous. Let $x \in B_{1}$ and $r_{n} \rightarrow r$ where each $r_{n}$ is rational and $r$ is real. Then $\left\|r_{n} x-r x\right\|_{1} \rightarrow 0$ and $\| r T(x)-T(r x)-$ $T\left(r_{n} x-r x\right) \| \rightarrow 0$. Hence $r T(x)-T(r x) \in S$. The first statement follows by a straightforward argument.

Let $y_{n} \in S, \quad\left\|w-y_{n}\right\| \rightarrow 0$. There exists, for each $n$, an element $z_{n} \in B_{1}$ such that $\left\|y_{n}-T\left(z_{n}\right)\right\|<n^{-1}$ and $\left\|z_{n}\right\|_{1}<n^{-1}$. Then $\left\|w-T\left(z_{n}\right)\right\| \rightarrow 0$ so that $w \in S$. Hence $S$ is closed in $B$. Since $x \in S$ and $r$ rational imply $r x \in S$ it follows that $S$ is a real linear manifold. To show that $S$ is an ideal in $B$ it is enough to show that $x y$ and $y x \in S$ for $x \in S$ and $y=T(z) \in T\left(B_{1}\right)$. This, however, is a simple matter. Suppose next that $\left\|x_{n}-x\right\|_{1} \rightarrow 0$ where each $x_{n} \in T^{-1}(S)$. For each $n$ there exists $y_{n} \in B_{1}$ such that $\left\|T\left(x_{n}\right)-T\left(y_{n}\right)\right\|<n^{-1}$ and $\left\|y_{n}\right\|_{1}<n^{-1}$. Then $\left\|x-\left(x_{n}-y_{n}\right)\right\|_{1} \rightarrow 0$ while 
$\left\|T(x)-T\left[x-\left(x_{n}-y_{n}\right)\right]\right\| \rightarrow 0$ whence $T(x) \leqq S$. Hence $T^{-1}(S)$ is closed. It is readily seen to be a two-sided ideal in $B_{1}$.

Let $B^{c}$ be the completion of $B$ where we use $\|x\|$ to denote the norm in $B^{c}$ and $\rho(x)$ the spectral radius there. To show that $s \in S$ is a topological divisor of zero in $B$ it is sufficient to show that it is one in $B^{c}$. Choose a sequence $\left\{x_{n}\right\}$ in $B_{1}$ such that $\left\|s-T\left(x_{n}\right)\right\| \rightarrow 0$ and $\left\|x_{n}\right\|_{1} \rightarrow 0$. If $B_{1}$ is a normed $Q$-algebra $s$ is the limit of quasi-regular elements of $B^{c}$ by Lemma 2.1. Hence so also is $\lambda s$ for any real $\lambda$. By the arguments of $[11 ; 621]$ it suffices to rule out the possibility that both $B^{c}$ has an identity 1 and that $s$ has a two-sided inverse in $B^{c}$.

Suppose this is the case. Let $S_{0}$ be the separating set for $T$ considered as a mapping of $B_{1}$ into $B^{c}$. Clearly $S \subset S_{0}$. Then as $S_{0}$ is an ideal in $B^{c}, S_{0}=B^{c}$ and $1 \in S_{0}$. There exists a sequence $\left\{u_{n}\right\}$ in $B_{1}$ such that $\left\|1-T\left(u_{n}\right)\right\| \rightarrow 0$ and $\left\|u_{n}\right\|_{1} \rightarrow 0$. Since $1-T\left(u_{n}\right)$ and $T\left(u_{n}\right)$ permute we have by Lemma 2.1,

$$
1=\rho(1) \leqq \rho\left(1-T\left(u_{n}\right)\right)+\rho\left(T\left(u_{n}\right)\right) \leqq\left\|1-T\left(u_{n}\right)\right\|+\rho\left(u_{n} \mid B_{1}\right) \rightarrow 0
$$

This contradication completes the argument.

If $B_{1}$ and $B$ are Banach algebras, by the closed graph theorem [2 ; 41] $S=(0)$ will imply that $T$ is continuous. In every case $S=(0)$ will imply real-homogeneity for $T$ and the closure of $T^{-1}(0)$.

3.2. Lemma. Let $B_{1}$ be a normed Q-algebra and $B$ be semi-simple with minimal one-sided ideals. Suppose that there exists a minimal onesided ideal $I$ of $B_{1}$ such that $T\left(B_{1}\right) \cap I \neq 0$. Then $S \cap I=(0)$.

We consider the case where $I$ is a right ideal and $T$ is a homomorphism. The other cases follow by the reasoning employed. Set $I_{1}=$ $T^{-1}(I) . \quad I_{1}$ is a right (ring) ideal of $B_{1}$. Let $I=j B, j^{2}=j$ and consider $x_{0} \in I_{1}$ where $T\left(x_{0}\right)=j v \neq 0$. By the semi-simplicity of $B, j v B \neq(0)$ and, as $j B$ is minimal, $j v B=j B$. Then $j v T\left(B_{1}\right)$ is dense in $I$. It follows that $T\left(I_{1}^{2}\right) \neq(0)$ for otherwise $\left[j v T\left(B_{1}\right)\right]^{2}=(0)$ and $I^{2}=(0)$. Select $x \in I_{1}, T(x)=$ $j w \neq 0$ and $T\left(x^{2}\right) \neq 0$. Let $R$ be the set of elements $y$ in $B$ for which $j y \in T\left(I_{1}\right)$. As observed, $j R$ is dense in $j B$. Hence $j R j$ is dense in $j B j$. But $j B j$ is a normed division algebra and therefore, by the GelfandMazur theorem, finite-dimensional in $B$. Thus $j R j=j B j$. There exists $z \in R$ such that $j z j w j=j w j z j=j$. For some $x_{1} \in I_{1}, T\left(x_{1}\right) j=j z j$. Then $T\left(x_{1} x\right)=j z j w=T\left(\left(x_{1} x\right)^{2}\right)$. Set $j z j w=h$ and $x_{1} x=u$. Then $h$ is a non-zero idempotent in $I \cap T\left(B_{1}\right)$. Clearly $h B=I$ so that $h B h$ is a division algebra hence isomorphic to the reals, complexes or quaternions.

We show that $h \notin S$. For suppose otherwise. Then there exists a sequence $\left\{y_{n}\right\}$ in $B_{1}$ such that $\left\|h-T\left(y_{n}\right)\right\| \rightarrow 0$ and $\left\|y_{n}\right\|_{1} \rightarrow 0$. Thus $\left\|u y_{n} u\right\|_{1} \rightarrow 0$ and $\left\|h-T\left(u y_{n} u\right)\right\| \rightarrow 0$. By Lemma 2.2 and the fact that $h B h$ is the reals, complexes or quaternions, $\left\|h T\left(y_{n}\right) h\right\| \rightarrow 0$, This is a 
contradiction as $h \neq 0$. Now $S \cap I$ is a right ideal of $B, S \cap I \neq I$. Since $I$ is minimal, $S \cap I=(0)$.

3.3. Theorem. Let $B_{1}$ be a normed Q-algebra and $B$ be primitive with minimal one-sided ideals. If $T\left(B_{1}\right) \cap I \neq(0)$ for a minimal one-sided ideal $I$ of $B$ then $T$ is closed and real-homogeneous.

Let $K$ be the socle of $B$. If $S \neq(0)$ then $K \subset S$ by [6;75]. Then $I \subset S$ which is impossible by Lemma 3.2.

3.4. CoRollary. Let $B$ be any subalgebra of $(\mathfrak{F}(\mathfrak{X})$ closed in the uniform norm $\|T\|$ where $B \supset \mathfrak{F}(\mathfrak{X})$. Let $\|T\|_{1}$ be any normed algebra norm for $B$ such that the completion $B^{c}$ of $B$ in this norm is primitive. Then the two norms are equivalent.

By Theorem 2.6 and Lemma 2.5, $B$ is a $Q$-algebra in the norm $\|T\|_{1}$. By Theorem 2.3, there exists $c>0$ such that $\|T\| \leqq c\|T\|_{1}, T \in B$. Consider the embedding mapping $I$ of $B$ (with norm $\|T\|$ ) into $B^{c}$. $B$ is a primitive algebra with a minimal right ideal $J B, J^{2}=J$. Then $I(J) I(B) I(J)$ a normed division algebra and, by the Gelfand-Mazur theorm, closed in $B^{c}$. Since $I(J)$ is an idempotent, its closure in $B^{c}$ is $I(J) B^{c} I(J)$. Therefore $I(J) B^{c}$ is a minimal right ideal of $B^{c}$. From Theorem 3.3, I is closed. The closed graph theorem $[2 ; 41]$ shows that $I$ is continuous. Hence there exists $c_{1}>0$ such that $\|T\|_{1} \leqq c_{1}\|T\|, T \in B$.

3.5. THEOREM. Let $B_{1}$ and $B$ be normed Q-algebras. Then $S$ is contained in the Brown-McCoy radical of $B$. If $B$ is strongly semisimple then $T$ is closed and real-homogeneous.

The Brown-McCoy radical [5] coincides with the intersection of the regular maximal two-sided ideals of $B$. Let $M$ be such an ideal of $B$. Since $B$ is a normed $Q$-algebra, $M$ is closed. Let $\pi$ be the natural homomorphism of $B$ onto $B / M$. Since $T\left(B_{1}\right)$ is dense in $B$, then $\pi T\left(B_{1}\right)$ is dense in $B / M$. Also $\rho[\pi T(x)] \leqq \rho[T(x)] \leqq \rho(x), x \in B_{1}$. Hence our theory applies to the mapping $\pi T$.

Let $S_{0}$ be the separating set for $\pi T$. Since $B / M$ is simple with an identity, $S_{0}=(0)$ by Lemma 3.1. Let $y \in S$, $\left\|x_{n}\right\|_{1} \rightarrow 0,\left\|y-T\left(x_{n}\right)\right\| \rightarrow 0$. Then $\left\|\pi(y)-\pi T\left(x_{n}\right)\right\| \rightarrow 0$ or $\pi(y) \in S_{0}$. Therefore $S \subset M . \quad B$ is called strongly semi-simple if its Brown-McCoy radical is (0).

3.6. THEOREM. Let $B_{1}$ and $B$ be semi-simple normed Q-algebras where $B_{1}$ has a dense socle $K$ and $B$ has an identity Let $T$ be real-linear. Then Tis closed.

Let $P$ be a primitive ideal of $B$ and $\pi$ be the natural homomorphism of $B$ onto $B / P$. Since $B$ is a $Q$-algebra then $P$ is closed, $\pi$ is continuous and $\pi T\left(B_{1}\right)$ is dense in $B / P$. Let $S_{0}$ be the separating set for $\pi T$ 
as a mapping of $B_{1}$ into $B / P$. We show first that $T(K) \subset P$ is impossible. Suppose $T(K) \subset P$. Since $K \subset(\pi T)^{-1}\left(S_{0}\right)$, by Lemma 3.1 we have $B_{1}=(\pi T)^{-1}\left(S_{0}\right)$ and $S_{0}=B / P$. Since $B / P$ has an identity this is contrary to Lemma 3.1. Hence there exists a minimal right ideal $j B_{1}$ of $B_{1}, j^{2}=j$ such that $T(j) \notin P$. Set $\pi T(j)=u, \pi T\left(B_{1}\right)=B_{2} . \quad \pi T$ is an isomorphism or anti-isomorphism of the division algebra $j B_{1} j$ onto $u B_{2} u$. Hence $u B_{2} u$ is a normed division algebra and thus, by the Gelfand-Mazur theorem closed in $B / P$. Since $u$ is an idempotent, $u(B / P)$ is a minimal right ideal of $B / P$. By Theorem 3.3, $\pi T$ is closed from which we obtain $S \subset P$. Since $B$ is semi-simple, $S=(0)$.

3.7. THEOREM. Let $B_{1}$ be a normed Q-algebra and $B$ semi-simple where either $B$ is a modular annihilator algebra or has dense socle. If $T\left(B_{1}\right)$ contains the socle of $B$ then $T$ is closed and real-homogeneous.

By Lemma 3.2, $S \cap I=(0)$ for every minimal one-sided ideal of $B$. Let $I$ be a minimal right ideal. Then $S I=(0)$. Thus $S$ annihilates the socle. It follows (see the proof of Lemma 2.8) that $S=(0)$ in the first case. In the second case we have $S^{2}=(0)$ and $S=(0)$ by semi-simplicity.

Consider further a semi-simple normed modular annihilator algebra $B$. $B$ is a permanent $Q$-algebra by Lemma 2.5 and 2.8. From Theorem 3.7 we see that any algebraic homomorphism or anti-homomorphism of $B$ onto $B$ is closed no matter which two norms are used for $B$.

Let $B$ be a real normed algebra. By an involution on $B$ we mean a mapping $x \rightarrow x^{*}$ of $B$ onto $B$ which is a real-linear automorphism or anti-automorphism of period two. Let $H(K)$ be the set of self-adjoint (skew) elements of $B$ with respect to the involution $x \rightarrow x^{*} . \quad B$ is the direct sum $H \oplus K$ of the linear manifolds $H$ and $K$.

The mapping $x \rightarrow x^{*}$ of $B$ onto $B$ is subject to the above analysis. Here $S$ is the set of all $x \in B$ for which there exists a sequence $\left\{x_{n}\right\}$ in $B$ with $\left\|x_{n}\right\| \rightarrow 0$ and $\left\|x-x_{n}^{*}\right\| \rightarrow 0$.

3.8. Lemma. $S=\bar{H} \cap \bar{K} . \quad S=(0)$ if and only if $H$ and $K$ are closed. Let $w \in S$. Then there exist sequences $\left\{h_{n}\right\}$ and $\left\{k_{n}\right\}$ in $H$ and $K$ respectively such that $\left\|w-\left(h_{n}-k_{n}\right)\right\| \rightarrow 0$ and $\left\|h_{n}+k_{n}\right\| \rightarrow 0$. Therefore $\left\|w-2 h_{n}\right\| \rightarrow 0$ and $\left\|w+2 k_{n}\right\| \rightarrow 0$ so $w \in \bar{H} \cap \bar{K}$. Conversely suppose that $\left\|z-h_{n}\right\| \rightarrow 0, \quad\left\|z-k_{n}\right\| \rightarrow 0$ where each $h_{n} \in H, \quad k_{n} \in K$. Then $\left\|z-\left(h_{n}+k_{n}\right) / 2\right\|$ $\rightarrow 0$ and $\left\|\left(h_{n}-k_{n}\right) / 2\right\| \rightarrow 0$ and $z \in S$.

If $H$ and $K$ are closed, clearly $S=(0)$. Suppose $S=(0)$. Let $h_{n} \rightarrow$ $u+v$ where $h_{n} \in H, u \in H$ and $v \in K$. Then $h_{n}-u \rightarrow v$ and $v \in \bar{H} \cap \bar{K}$. Then $v=0$ and $H$ is closed. Similarly $K$ is closed.

Let $B$ be a semi-simple normed annihilator algebra, for example an $H^{*}$-algebra. Then it follows from the above that $H$ and $K$ are closed in $B$ for any involution on $B$ and any normed algebra norm on $B$. For 
$B^{*}$-algebras we have been able to show only the following weaker result.

3.9. THEOREM. Let $B$ be a $B^{*}$-algebra with $H(K)$ as the set of selfadjoint (skew) elements in the defining involution for $B$. Then $H$ and $K$ are closed in any normed algebra norm topology for $B$.

$B$ has the spectral extension property [13] and is therefore a permanent $Q$-algebra by Lemma 2.5 . The arguments of $[14 ; \S 3]$ can be adapted to show that $H$ and $K$ are closed in any given normed algebra norm $\|x\|_{1}$.

\section{REFERENCES}

1. B. H. Arnold, Rings of operators on vector spaces, Ann. of Math., 45 (1944), 24-49.

2. S. Banach, Theorie des operations lineairs, Monog. Mat., Warsaw, 1932.

3. F. F. Bonsall, A minimal property of the norm in some Banach algebras, J. London, Math. Soc., 29 (1954), 156-164.

4. F. F. Bonsall and A. W. Goldie, Annihilator algebras, Proc. London Math. Soc., 4 (1954), 154-167.

5. B. Brown and N. H. McCoy, Radicals and subdirect sums, Amer. J. Math., 69 (1947), 46-58.

6. N. Jacobson, Structure of rings, Amer. Math. Soc. Colloq. Publ. vol. 37, Providence, 1956.

7. I. Kaplansky, Dual rings, Ann. of Math., 49 (1948), 689-701.

8. I. Kaplansky, Normed algebras, Duke Math, J., 16 (1949), 399-418.

9. I. Kaplansky, Ring isomorphisms of Banach algebras, Can. J. Math., 6 (1954), 374-381.

10. E. A. Michael, Locally multiplicatively-complex topological algebras, Mem. Amer. Math. Soc. no. 11 (1952).

11. C. E. Rickart, The uniqueness of norm problem in Banach algebras, Ann. of Math. 51 (1950), 615-628.

12. C. E. Rickart, Representation of certain Banach algebras, Duke Math. J., 18 (1951), 27-39.

13. C. E. Rickart, On spectral permanence for certain Banach algebras, Proc. Amer. Math. Soc., 4 (1953), 191-196.

14. B. Yood, Topological properties of homomorphisms between Banach algebras, Amer.

J. Math., 76 (1954), 155-167.

UNIVERSITY OF OREGON 



\section{PACIFIC JOURNAL OF MATHEMATICS}

\section{EDITORS}

\section{H. L. Royden}

Stanford University

Stanford, California

\section{R. A. Beaumont}

University of Washington

Seattle 5 , Washington

\author{
A. L. Whiteman
}

University of Southern California

Los Angeles 7, California

E. G. Straus

University of California

Los Angeles 24, California

\section{ASSOCIATE EDITORS}
E. F. BECKENBACH
A. HORN
L. NACHBIN
G. SZEKERES
C. E. BURGESS
V. GANAPATHY IYER
I. NIVEN
F. WOLF
M. HALL
R. D. JAMES
T. G. OSTROM
E. HEWITT
M. S. KNEBELMAN
M. M. SCHIFFER
K. YOSIDA

\section{SUPPORTING INSTITUTIONS}

\author{
UNIVERSITY OF BRITISH COLUMBIA \\ CALIFORNIA INSTITUTE OF TECHNOLOGY \\ UNIVERSITY OF CALIFORNIA \\ MONTANA STATE UNIVERSITY \\ UNIVERSITY OF NEVADA \\ OREGON STATE COLLEGE \\ UNIVERSITY OF OREGON \\ UNIVERSITY OF SOUTHERN CALIFORNIA
}

\author{
STANFORD UNIVERSITY \\ UNIVERSITY OF UTAH \\ WASHINGTON STATE COLLEGE \\ UNIVERSITY OF WASHINGTON \\ * * * * \\ AMERICAN MATHEMATICAL SOCIETY \\ CALIFORNIA RESEARCH CORPORATION \\ HUGHES AIRCRAFT COMPANY \\ THE RAMO-WOOLDRIDGE CORPORATION
}

Mathematical papers intended for publication in the Pacific Journal of Mathematics should be typewritten (double spaced), and the author should keep a complete copy. Manuscripts may be sent to any of the editors. All other communications to the editors should be addressed to the managing editor, E. G. Straus at the University of California, Los Angeles 24, California.

50 reprints per author of each article are furnished free of charge; additional copies may be obtained at cost in multiples of 50 .

The Pacific Journal of Mathematics is published quarterly, in March, June, September, and December. The price per volume (4 numbers) is $\$ 12.00$; single issues, $\$ 3.50$. Back numbers are available. Special price to individual faculty members of supporting institutions and to individual members of the American Mathematical Society: $\$ 4.00$ per volume; single issues, $\$ 1.25$.

Subscriptions, orders for back numbers, and changes of address should be sent to Pacific Journal of Mathematics, 2120 Oxford Street, Berkeley 4, California.

Printed at Kokusai Bunken Insatsusha (International Academic Printing Co., I.td.), No. 10, 1-chome, Fujimi-cho, Chiyoda-ku, Tokyo, Japan.

PUBLISHED BY PACIFIC JOURNAL OF MATHEMATICS, A NON-PROFIT CORPORATION

The Supporting Institutions listed above contribute to the cost of publication of this Journal, but they are not owners or publishers and have no responsibility for its content or policies. 


\section{Pacific Journal of Mathematics}

\section{Vol. 8, No. 2 \\ April, 1958}

John Herbert Barrett, Second order complex differential equations with a real independent variable ............................ 187

Avner Friedman, Remarks on the maximum principle for parabolic equations and its applications ......................... 201

Richard Robinson Goldberg, An inversion of the Stieltjes transform ....... 213

Olavi Hellman, On the periodicity of the solution of a certain nonlinear integral equation .................................. 219

Gilbert Helmberg, A theorem on equidistribution on compact groups...... 227

Lloyd Kenneth Jackson, Subfunctions and the Dirichlet problem ......... 243

Naoki Kimura, The structure of idempotent semigroups. I ............ 257

Stephen Kulik, A method of approximating the complex roots of equations........................................ 277

Ancel Clyde Mewborn, A note on a paper of L. Guttman.............. 283

Zeev Nehari, On the principal frequency of a membrane ............ 285

G. Pólya and I. J. Schoenberg, Remarks on de la Vallée Poussin means and convex conformal maps of the circle ...................... 295

B. M. Stewart, Asymmetry of a plane convex set with respect to its centroid .......................................... 335

Hans F. Weinberger, Lower bounds for higher eigenvalues by finite difference methods

Edwin Weiss and Neal Zierler, Locally compact division rings ......... 369

Bertram Yood, Homomorphisms on normed algebras ................. 373 